\title{
Armut infolge öffentlicher Vernachlässigung
}

\section{Arme Eltern und arme Städte verhalten sich ähnlich ...}

\author{
Armin Kuphal
}

Der Soziologe Dr. Armin Kuphal ist wissenschaftlicher Mitarbeiter der Universität des Saarlandes und Vorsitzender des Paritätischen Bildungswerkes RheinlandPfalz/Saarland. Er war Mitautor des ersten Armutsberichtes des Paritätischen Wohlfahrtsverbandes im Jahre 1989.

E-Mail a.kuphal@mx.uni-saarland.de
Es gibt eine Parallele zwischen dem Handeln armer Eltern und dem Handeln einer armen öffentlichen Hand: Bei beiden ist die Gefahr groß, dass sie ibre Kinder vernachlässigen. Denn nicht nur die Eltern, auch die Gemeinschaft ist verantwortlich für das Recht junger Menschen auf Förderung ihrer Entwicklung und auf Erziehung zu einer eigenverantwortlichen und gemeinschaftsfähigen Persönlichkeit, wie es das Kinder- und Jugendhilfegesetz verlangt.

Vor einiger Zeit ging eine richtig gute Meldung durch die Presse. Sie stammte vom Deutschen Jugendinstitut (DJI) in München und sie bezog sich auf die Ergebnisse einer repräsentativen Befragung von Kindern, dem »Kinderpanel«. Auf die Kurzformel gebracht hieß die gute Nachricht: "Kinder geben Schule und Familie gute Noten« oder: »Die meisten Kinder sind zufrieden.«

Das emotionale Klima, so das Institut, ist in den meisten Familien recht positiv. Die meisten Kinder fühlen sich in Familie und Freundeskreis wohl. Fast alle 8- bis 9-Jährigen finden sich selbst »okay « und sind meist gut gelaunt. Sie erleben sich sozial und kognitiv als sehr aufgeschlossen, probieren gerne Neues aus, lernen gerne neue Kinder kennen, haben viele Ideen und glauben, sich gut in andere hinein versetzen zu können. Auch die befragten Eltern beschreiben ihre Kinder ähnlich positiv.

Für viele Kinder seien jedoch auch Kummer und Enttäuschung eine normale Erfahrung: Drei Viertel der 8- bis 9Jährigen seien manchmal traurig oder ängstlich, die Hälfte fühlte sich manchmal allein usw. Die Eltern nehmen diese Gefühle bei ihren Kindern nicht so häufig wahr, ganz im Gegensatz zu Ausdrucksformen wie Wut auf andere, die ein Viertel der Kinder äußert, oder Lust am Raufen, die ein Drittel der Jungen und jedes sechste Mädchen äußern. Als zentrales Ergebnis des DJI-Kinderpanels könne festgehalten werden, so die Forscher in ei- ner Verlautbarung: »Ein positives Familienklima und eine günstige Wohnumgebung gehen mit einem positiveren Selbstbild und größerer Aufgeschlossenheit der Kinder einher."

Sowohl die gestellten Fragen wie die ermittelten Antwortverteilungen scheinen mir nicht sehr erhellend für jemanden, der mit trennscharfen Fragen praxisrelevante Unterscheidungen finden möchte. Kinder sind tatsächlich manchmal traurig und manchmal ängstlich - Erwachsene übrigens auch.

Und wo bleibt jene Wirklichkeit, mit der Fachkräfte der Sozialen Arbeit tagtäglich zu tun haben? Könnte es sein, dass wir Profis zu selektiv wahrnehmen und in professioneller Überempfindlichkeit überall nur noch »Probleme " wahrnehmen und die guten Seiten übersehen? Ich glaube nicht. Ich nehme vielmehr an, dass das Instrumentarium des Kinderpanels zu undifferenziert ist für die Lebenswelt von Kindern, die wir als »arm « bezeichnen. Bemerkenswert ist, dass die Daten, die die Forscher aus Beobachtungen gewonnen haben, zu einem weniger positiven Bild führen: Es wird festgestellt, das Wohnumfeld sei bei einem Drittel der Kinder nicht kindgerecht, also: die elterliche Wohnung zu klein und schlecht ausgestattet, die nähere Umgebung bietet wenig Spielmöglichkeiten, das Umfeld hat eine hohe Verkehrsbelastung etc.

\section{Armut als Systemzustand}

Erst mit der relativ jungen Idee der sozialen Gleichheit bekam der Armutsbegriff jene Brisanz, die wir heute empfinden. Im Mittelalter war »der arme Mann« nur eine soziale Kategorie für die der Leibherrschaft des Grundherrn unterworfenen Untertanen. Heute ist »Armut « ein Schwellenbegriff, der einen Umschlag von einer Quantität in eine andere Qualität mit besonderem Handlungsbedarf markiert. 
Wenn wir heute sagen »Es herrscht Armut ", dann bezeichnen wir damit nicht die individuelle Lage eines Menschen, sondern den Zustand eines sozialen Systems. Das Augenmerk ist mithin nicht nur darauf zu richten, ob die Zahl der (Einkommens-) Armen zunimmt und ob die Armen ärmer werden. Es ist auch und verstärkt darauf zu achten, ob und wie Armut sich systemisch verdichtet.

Wenn heute Armut herrscht, dann in einem anderen Sinne als noch vor zwanzig Jahren, als der erste bundesweite Armutsbericht des Paritätischen Wohlfahrtsverbandes für Aufsehen sorgte. Die Gemeinwesenarbeit in Westdeutschland war von ihrem Beginn um 1970 bis in die 1980er Jahre damit beschäftigt, Menschen aus Baracken und Schlichtwohnungen am Rande der Stadt zu holen, ihnen zu normalen Wohnungen zu verhelfen, zu normaler Schulbildung und nicht zuletzt zu normalen Sozialleistungen, indem sie die Menschen in ihren sozialen Rechten stärkten, lahme Ämter und Dienste auf Trapp brachten und zu Übergriffen neigende Behörden in ihre Schranken wiesen.

Die Baracken und Schlichtbauten gibt es längst nicht mehr und die Wohnquartiere des sozialen Wohnungsbaus sehen heute anders aus als damals. Damals war es unter Kindern im Stadtteil geläufig, sich »Assi-Barackler « zu beschimpfen. Dieses nur ein Indiz dafür, wie virulent die Aussonderung im kollektiven Bewusstsein vorhanden war. Was rufen sie heute, wo verlaufen heute die Linien der verletzenden Unterscheidung?

Die neue Armut ist weniger dramatisch, ist weniger offenkundig, ist gestaltloser. Das macht es zum Beispiel schwer, das Thema Armut mediengerecht darzustellen - das Fernsehen will eben hungernde Kinder im Bild haben. Man hat Mühe, die neue Erscheinungsform der Armut auf den systemischen Begriff zu bringen. Was ist es, was ist anders? Wenn es nicht mehr die Verhältnisse in den Baracken sind - was macht Kinder heute arm? Welche Bedingungen sind so nachteilig, dass wir sagen "es herrscht Armut «? Was drückt Menschen zu Boden, was führt dazu, dass diese Lebenslage auf Kinder weitervererbt wird? Ich gebe vier Hinweise: die kapitalistische Großwetterlage, der demonstrative Wohlstand, die Kultur, die Individualisierung.

\section{Die kapitalistische Großwetterlage}

»Die Armut kommt von der Poverté«, hieß es einst in Preußen. Gemeint war die Magerkeit der märkischen Sandböden. Heute ist es die Magerkeit des Arbeitsmarktes. Weil bei gesättigten Märkten die Renditen sinken, gibt es seitens des Kapitals zwei große Strategien:

- Die eine Möglichkeit, die Renditen zu halten besteht darin, Steuern zu sparen. Deshalb werden wir mit den Mitteln des modernen Agenda Settings damit beschallt, die Steuern müssten trotz der Finanznot des Staates gesenkt werden. Das Argument: Konkurrenz mit dem Ausland und Anschiebung der Wirtschaft. Der dadurch entstehende Einnahmeausfall aufseiten des Steuerstaates tragen die, die sich nicht wehren können.

- Strategie Nummer zwei besteht darin, Leistungen zu übernehmen, die bisher der Staat vorgehalten hat, also Privatisierung mit nachfolgender Rationalisierung und das heißt: Entlassungen. Argument: Privatunternehmen wirtschaften besser als der Staat. Am härtesten von den Gebührenerhöhungen werden dann die Armen getroffen.

Arme Eltern können ihren Kindern keine guten Zeiten in Aussicht stellen. Auch der hie und da mal mögliche Euro für »Luxus « ist weggefallen, weil in $\mathrm{Zu}$ sammenhang mit Hartz IV die Kontrollen der Arbeitsämter inzwischen so dicht geworden, dass gelegentliches Geld nebenher sehr erschwert wurde.

\section{Mehr demonstrativer Wohlstand}

Im allgemeinen Sinne herrscht Armut, weil auf der anderen Seite unverhüllter sichtbarer angeberischer protziger Wohlstand herrscht; nicht nur im Sinne der systematischen Privilegierung, dass Geld zu Geld kommt, sondern auch im ganz konkreten Sinne, dass sich in einer kapitalistischen Grundordnung das Geld überall, im Großen wie im Kleinen, durchsetzt.

Hierher passt die Theorie des demonstrativen Konsums. Die neue Spaltung der Gesellschaft in ein Oben und Unten zeigt sich danach verstärkt im Konsum, ja der Markt spalte sich mehr und mehr in ein Segment für Wohlhabende und in ein
Arme-Leute-Segment, während Sortimenter für den mittleren Bedarf - so auch die Innenstadtkaufhäuser - in Not geraten. Ich teile die These einer Segmentierung des Marktes als wirtschaftliche und soziale Tatsache, nicht aber die, dass der Konsum bei Aldi, Lidl, Schlecker, Plus usw. im Bewusstsein der Kunden diskriminierend sei.

Geringverdiener werden durch hohe Preise nicht aus dem Feinkostladen und der Edelmarken-Boutique verdrängt da waren sie noch nie als Kunden akkreditiert. Deutlicher als in der Vergangenheit zeigt sich, dass Minderbemittelte aus der städtischen Öffentlichkeit verdrängt werden - durch die Preise und zur Not durch spezielles Personal. Wenn ich auf dem Schlossplatz in Saarbrücken oder einem anderen sonnigen Ort der Stadt ein Bier trinke und aus meinem Einkommen dafür 3,50 Euro zahle, dann müssen Sozialleistungsbezieher und Geringverdiener in Relation zu ihrem Einkommen zehn Euro dafür hinlegen - und dann ist klar, dass bestimmte Orte und Veranstaltungen in der Stadt für bestimmte Menschen und insbesondere für Geringverdiener mit Kindern tabu sind. Wenn zur Finanzierung öffentlicher Dienstleistungen immer mehr und immer höhere Gebühren erhoben werden, entfallen Besuche in Einrichtungen, die Kinder um ihretwillen nutzen sollten. Ich denke vor allem an das Schwimmbad: Jedes dritte Kind lernt nicht mehr schwimmen.

Wenn soziale Teilhabe und wichtige Fertigkeiten nur noch über hohe Preise zu haben sind, die Geringverdiener nicht mehr zahlen können, ist dies eine Form systematischer Ausschließung. Zu solcher Ausschließung zähle ich, wenn armen Menschen die Einrichtung eines Girokontos verweigert wird.

\section{Von der Kultur der Armen zu einer armen Kultur}

Die stärkste Determinante der Armut liegt in der Bildung - im engen Sinne der Ausbildung und im weiteren Sinne von Kultur. Während der Faktor Bildung in modernen Industrie und Dienstleistungsgesellschaften immer wichtiger wird, verliert er in der armen Bevölkerung immer mehr an Wert. Dafür gibt es viele Gründe - ich nenne vier: 
- Erstens: Schon die Pflichtschule schafft es bis auf löbliche Ausnahmen nicht, die von Kindern mitgebrachten Benachteiligungen auszugleichen - danach sind erst weiterführenden Schulen und Universitäten erst recht kein Thema mehr. Dieses Versagen war in den 1960er Jahren einmal Thema: »Kompensatorische Erziehung " war damals das Stichwort für eine soziale Korrektur der Schule. Dieser kritische Impuls ist verschwunden. Die durch PISA angestoßene Diskussion um die Qualität der deutschen Schule kam denn ja auch nicht durch unmittelbare Kritik an der deutschen Schule oder durch eine positive Vision über die Zukunft der Schule auf die politische Tagesordnung, sondern durch das grottenschlechte Abschneiden im internationalen Vergleich. Deutschland auf der Höhe von Entwicklungsländern - das hat wehgetan, während der eigentlich beanstandete Sachverhalt bildungspolitisch nicht mehr wahrgenommen wurde. Die in dem Bildungssystem benachteiligten eigentlich wollen, verlieren die Ziele ihre Geltung. Wir erleben junge Leute, die in Sozialhilfe oder Arbeitslosengeld eine verlässliche Rente sehen und in realistischer Einschätzung ihrer Fähigkeiten und des Arbeitsmarktes keinen wirksamen Wunsch entwickeln, dort herauszukommen. Wenn die gesamte soziale Umgebung so betroffen ist, wird diese Existenz zur sozialen Normalität.

- Drittens: Ohne Beruf und Erwerbsarbeit fehlt schließlich ein Lehrmeister für eine geordnete rationale Lebensführung. Motive zur Eigenwirtschaft, so denn in Mietwohnungen überhaupt Möglichkeiten dafür gegeben sind, verkümmern ebenso: Armut durch doppelte Arbeitslosigkeit - erstens durch fehlende Erwerbsarbeit und zweitens durch die fehlende Eigenarbeit. Mangels wirklicher Praxis schwinden die Fähigkeiten, sich selber zu organisieren, miteinander $\mathrm{zu}$ kooperieren und sich untereinander zu vernetzen. Dieses Defizit an Selbstorganisation wird selbst von einer Seite bescheinigt, die eigent-

\section{"Schwache Eltern schicken schwache Kinder ins Leben"}

Kinder und Eltern können in ihrer Art keinen Einfluss auf Schule ausüben und sie wollen deshalb auch nicht. Wenn arme Leute die Schule nicht schaffen, dann legen sie sich nicht mir ihr an, sondern sie meiden sie.

- Zweitens: Ohne Bezug auf eine Arbeit, die Lebensunterhalt und Sinnstiftung gibt, verlieren schulischer Wissenserwerb, Ausbildung, Bildung und Selbstorganisation an Bedeutung. Dabei gibt es wahrscheinlich einen Kumulierungseffekt, wenn von der einen Generation auf die nächste die Erfahrung gemacht wird, dass auf dem Arbeitsmarkt für gering Qualifizierte keine reellen Chancen bestehen. Ohne die stabile Erfahrung, dass durch eigene Anstrengung und Ausdauer ein Erfolg erzielt wird, findet kein positives Lernen statt. Wenn es im Ergebnis egal ist, ob ich mich anstrenge oder nicht, verkümmert die eigene Motivation. Wenn Menschen chronisch nicht erreichen, was sie lich froh darüber sein könnte, nämlich der Polizei: Organisierte Kriminalität könne es in bestimmten Wohngebieten deshalb nicht geben, weil die Jungs dort überhaupt nicht in der Lage seien, sich zu organisieren.

- Und schließlich viertens ist zu erwähnen, dass das Volk zur Ablenkung von seinem Elend, wie Karl Marx schon bemerkte, gerne zum Opium greift - zu Betäubungsmitteln aller Art, zu denen auch das Fernsehen zählt.

Wir sind noch bei der Frage, was Armut determiniert; dazu gehört das Risiko der Individualisierung und Kontrollverlust:

\section{Jeder für sich und niemand für alle}

Eine in den Sozialwissenschaften akzeptierte Theorie über die Moderne ist, dass wir in einer gewaltigen Welle der In- dividualisierung begriffen sind. Traditionelle Einbindungen gehen verloren - ein jeder steht als Kapitän auf seinem eigenen Lebensschiff und muss seinen eigenen Kurs finden, wo man früher auf großen Schiffen mitgenommen wurde. Diese Bedingungen sind für nicht wenige von Vorteil, für viele bergen sie aber große Risiken - bei der Lebensplanung und bei der Deutung des eigenen Elends, wenn es schief geht.

Menschen halten viel aus, wenn Hoffnung auf Erlösung besteht. Ich sehe kaum noch Institutionen, die noch in der Lage wären, Hoffnungen zu machen. Die letzte Hoffnung der kleinen Leute, die SPD, ist wohl schon vor längerer Zeit gestorben, sie wird nur jetzt erst beerdigt. Davor haben viele andere Institutionen ihren Ruf verloren, wie Felsen in der Brandung zu stehen, voran die Rentenversicherung. Die Armut von heute geht auf die Familie - wer heute in finanziell engen Verhältnissen Kinder erziehen muss, hat etwas nicht mehr, was die Generation davor noch hatte: eine gewisse politische Beachtung und die nicht ganz unbegründete Hoffnung, dass es besser wird.

\section{Kinderarmut als Folge der Armut der Eltern}

Man kann eine Parallele ziehen zwischen dem Handeln armer Eltern und dem Handeln einer armen Stadt: Bei beiden ist die Gefahr groß, dass sie ihre Kinder vernachlässigen.

Kinderarmut ist kein Sachverhalt für sich, sondern ist eine aus der Lebenslage der Eltern abgeleitete Existenz mit ihrer Not, ihren Mängeln, ihren Defiziten und der ihr anhaftenden Minderwertigkeit. Die Abhängigkeit von Kindern, ihre Empfindlichkeit für frühe Deprivation und die Nachhaltigkeit dieser negativen Erfahrung für das ganze Leben sind mit Gründe, weshalb Kinderarmut besonderer Aufmerksamkeit und besonderer Antworten bedarf.

Was in der Presse gerne als Kinderarmut dargestellt wird, hat in der Jugendhilfe einen präziseren Namen: Es heißt Kindesvernachlässigung und Kindesmisshandlung. Auch wenn grob vernachlässigte Kinder fast ausschließlich aus sozialen Randgruppen kommen, so ist die tatsächliche Vernachlässigung der Bedürfnisse von Kindern infolge der Ein- 
kommensarmut der Eltern eine fast als normal hingenommene Tatsache. Die Grenze zwischen den Einschränkungen, die Kinder in einer "normal armen ", also an der Armutsgrenze lebenden, Familie hinzunehmen haben und den Formen einfacher Kindesvernachlässigung ist fließend, sei es Fehlernährung durch minderwertige Nahrungsmittel, sei es fehlende Mittel für Kinder, wenn wesentliche Teile des Familienbudgets für Genussmittel wie Zigaretten und Alkohol ausgegeben werden.

Fließende Übergänge gibt es ebenfalls zu Formen der Kindesmisshandlung. Im Rahmen des grundgesetzlich garantierten Pflege- und Erziehungsrechtes der Eltern und im Rahmen dessen, was in unserer Gesellschaft als normal angesehen wird, gibt es erlaubte Zonen von Kindesvernachlässigung und Kindesmisshandlung. Beispielsweise zählen das Rauchen der Eltern - selbst bei Säuglingen - oder Fernsehen ohne jede Beschränkung rechtlich nicht als Kindesmisshandlung. Ich erwähne dies so ausdrücklich, weil hier gerne mit zweierlei Maß gemessen wird.

Der Zusammenhang mit dem fehlenden Geld liegt auf der Hand - aber daraus folgt nicht, dass durch mehr Geld alles gut wird. Wie richtig eingewendet wurde: Auch 100 Euro mehr werden in vielen Fällen nur dazu führen, dass dieses Mehr »systemimmanent « ausgegeben wird und nicht notwendig so, wie wir uns das zum Wohle der Kinder wünschen.

Dringend warnen möchte ich an der Stelle vor dem Satz, die beste Sozialpolitik sei eine gute Arbeitsmarktpolitik, sofern daraus gefolgert werden soll, die bestehenden sozialen Probleme werden automatisch gelöst, wenn der nächste Aufschwung kommt und die Arbeitslosigkeit abnimmt - und dass man genau daran arbeite ... Der Arbeitsmarkt wird sich zum einen nicht wieder berappeln und zum anderen haben sich viele Defizite armer Familien längst verselbständigt. Ihnen ist nur mit Geld nicht mehr beizukommen.

Wenn das System Familie unter den vorgenannten Bedingungen der Armut in seinen Erziehungsaufgaben faktisch überfordert wird, dann gibt es darauf eigentlich nur zwei Reaktionen. Entweder man übernimmt mit immer mehr Aufwand die ausfallenden Funktionen der Familie bis hin zur teuren Heimerziehung. Oder man versucht die Eltern und Familien darin zu stützen und zu stärken, dass sie ihre Auf- gaben gegenüber ihren Kindern erfüllen können.

Der erste Weg führt zu einem mehr und mehr unlösbaren Kapazitätsproblem aber es ist der einfachere Weg. Der zweite Weg ist komplizierter - er führt zu einem Problem der Umsteuerung der Institutionen, der Programme und der Menschen in der Jugendhilfe und aller anderen, die mit Sozialisationsaufgaben betraut sind. Als Sozialwissenschaftler und als Gemeinwesenarbeiter bin ich dennoch zutiefst davon überzeugt, dass es der richtige, weil weiterführende Weg ist.

Ich hoffe die Provokation gelingt mir, wenn ich mich übungshalber auf die Seite der Guten stelle und die Parallele zu vernachlässigenden Eltern ziehe:

\section{Kinderarmut als Folge der »normalen « öffentlichen Armut und als Folge öffentlicher Vernachlässigung}

Kindesvernachlässigung als unzureichende Pflege und Erziehung ist zunächst ein Tatbestand, der nach juristischer Logik den Erziehungsberechtigten zugerechnet wird. Das ist eine Einengung, welche der Wirklichkeit nicht gerecht wird. Neben den Eltern steht nämlich die staatliche Gemeinschaft, der Staat, die Stadt usw. in der erzieherischen Verantwortung gegenüber den Kindern. Von der Sache her müsste ein erweiterter Begriff der Kindesvernachlässigung auf das staatliche Handeln anzuwenden sein, auch wenn klar ist, dass der darin erfasste Tatbestand nicht justiziabel ist.

$\mathrm{Zu}$ erinnern ist hier an die seinerzeit sensationelle Erweiterung des Begriffs der Gewalt durch Johan Galtung: Wenn Kinder in einem Lande beispielsweise keine Schulausbildung erhalten, wird ihnen Gewalt angetan. Zur Unterscheidung von der sichtbaren personalen Gewalt spricht Galtung von »struktureller Gewalt «.

Wie wollen wir denn bewerten, wenn eine vorhandene Benachteiligung von Kindern in schulischen Einrichtungen nicht kompensiert, sondern durch chronische Beschämung vielmehr noch verstärkt wird? Wenn Kinder seelisch und körperlich an der Schule leiden und sich dem durch Fernbleiben zu entziehen versuchen - dann kommt eine kindzentrierte Betrachtung kaum an der Frage vorbei, ob hier nicht eine Form von psychischer Kin- desmisshandlung vorliegen könnte, der dringend durch Änderung der schulischen Bedingungen zu begegnen ist. Auch die Argumente, mit denen die öffentliche Unterlassungen entschuldigt werden, sind ähnlich denen, die vernachlässigende Eltern für sich in Anspruch nehmen: Kein Geld vorhanden, ist doch nicht so schlimm, andere tun es auch, es gibt keine Alternative, es hat mich keiner auf die dramatischen Folgen hingewiesen ...

Es bietet sich an, diese öffentliche Untätigkeit in Analogie zu der »normalen familialen Vernachlässigung « zu deuten: Man hat selber eine gewisse Ahnung, dass es nicht gut läuft, weiß es womöglich sogar, muss sich manchmal manches sagen lassen, sieht sich aber wegen vieler Hindernisse gleichwohl nicht in der Lage, etwas zu verändern. Kennen wir das nicht von armen Familien?

In dem Maße, wie Familien, soziale Milieus und Erziehungseinrichtungen an Erziehungskraft verlieren und schwache Eltern wiederum schwache Kinder ins Leben schicken, wächst die Belastung der lokalen Jugendhilfe als Kontroll- und Reparaturanstalt. Dies überlastet das Personal der Jugendämter und es überlastet die eh schon überlasteten kommunalen Kassen. Es ist zu befürchten, dass für die präventiven Ansätze, die sowieso nicht weit ausgebaut sind, die Mittel durch die akute Belastung noch weiter zurückgenommen werden. Denn präventive Leistungen sind nach der staatlichen Haushaltslogik meistens freiwillige Leistungen. Was mit denen geschieht, wissen wir. Wie wollen wir dieses staatliche Handeln bewerten? Wie sollen wir darauf reagieren?

\section{Wer gegen die öffentliche Vernachlässigung von Kindern intervenieren müsste}

Eltern werden kontrolliert, ob sie ihre Erziehungsaufgaben richtig wahrnehmen und im Falle familialer Vernachlässigung hat der Staat als Wächter zu intervenieren. Wer aber interveniert, wenn »der Staat « selber zulässt, dass seine Kinder nicht das kriegen, was ihnen zusteht wenn der Anspruch junger Menschen "auf Förderung ihrer Entwicklung und auf Erziehung zu einer eigenverantwortlichen und gemeinschaftsfähigen Persönlichkeit « ( $\mathbb{1} 1 \mathrm{KJHG})$ nicht erfüllt wird? Die Kinder können und werden nicht rea- 
gieren und ihre armen Eltern auch nicht; zumal sie, wie erwähnt, herkömmliche öffentliche Angebote der Bildung, der Erziehung, der Gesundheitspflege usw. eher meiden als sie in Anspruch zu nehmen.

Eine Strategie der 1968er-Studenten war, sich mit den eben entdeckten Armen am Rande der Städte verbünden zu wollen und von dort aus die Verhältnisse aufzumischen. Die »Randgruppenstrategie « war politische Romantik, ohne Basis und deshalb ohne Erfolg. Die linke Laufgemeinschaft Gysi/Lafontaine hat hingegen gute Chancen, über den Rand zurück in die Mitte zu kommen, wenn es ihr gelingt, die armen Nichtwähler zu mobilisieren und ihre Sympathisanten aufseiten der Sozialen Arbeit zu sensibilisieren. Ich kenne nicht wenige engagierte KollegInnen aus der Sozialen Arbeit, die bei allen Vorbehalten keine andere Wahl für eine Systemkorrektur im Sinne der Armen als diese sehen.

Wir Akteure der Jugendhilfe müssen wahrnehmen, was an dem bisherigen Kurs falsch war. Die prägnanteste Kritik stammt von dem Historiker Paul Nolte: Die Politik der Unterschichten und mar- ginalisierte Gruppen ist bei uns zu lange einem Konzept gefolgt, das man als » fürsorgliche Vernachlässigung « bezeichnen könnte. Einer vergleichsweise hohen materiellen Fürsorge steht eine Vernachlässigung in sozialer und kultureller Hinsicht gegenüber. Zugespitzt formuliert: Die Mehrheitsgesellschaft hat sich mit Geldzahlungen von den wirklichen Problemen am Rande der Gesellschaft freigekauft, sie hat sich ein gutes Gewissen erkauft, ohne Probleme zu lösen. Das funktioniert nicht mehr - für beide Seiten.

Die »staatliche Gemeinschaft « muss sich selbstkritisch prüfen, ob sie noch auf dem richtigen Weg ist, indem sie sich an den eigenen Zielen misst, statt abzuwarten, bis das Problem als Pflichtaufgabe wiederkommt oder je nach Kassenlage die Standards zu vermindern. Die Pädagogik hat u. a. mit PISA gezeigt, dass sie das Zeug zur objektiven korrigierenden Prüfung hat. Eine ähnliche Überprüfung steht für das System der Jugendhilfe aus. Ich meine dabei eine ernsthafte Systemevaluation mit dem Ziel der Verbesserung und nicht eine »Evaluation«, die nur als Vorwand für eh geplante Schließungen dieser oder jener Projekte herhalten soll.

Der stärkste Veränderungsdruck zur Bekämpfung der Kinderarmut könnte paradoxerweise aus der zunehmenden Finanznot der öffentlichen Jugendhilfe kommen. Vielleicht muss die herkömmliche Reparaturpolitik erst unbezahlbar teuer werden, bevor es Anstöße zu einem Handlungsprogramm gibt, welches den Namen Prävention oder Schaffung positiver Lebensbedingungen verdient und welches erfolgreicher und kostengünstiger wäre als der bisherige Betrieb. Der Auftrag dazu ist im Kinder- und Jugendhilfegesetz in klaren Worten längst gegeben es kommt darauf an, ihn tatsächlich umzusetzen.

Eine neue Strategie beruhte letztlich darauf, dass sich in einem überschaubaren Sozialraum die Menschen, die mit der Erziehung von Kindern befasst sind, sich als Teilhaber eines gemeinsamen positiven Programms zur Förderung von Familien und insbesondere zur Sozialisation von Kindern verstehen und in diesem Sinne miteinander kooperieren - generell und konkret von Fall zu Fall.

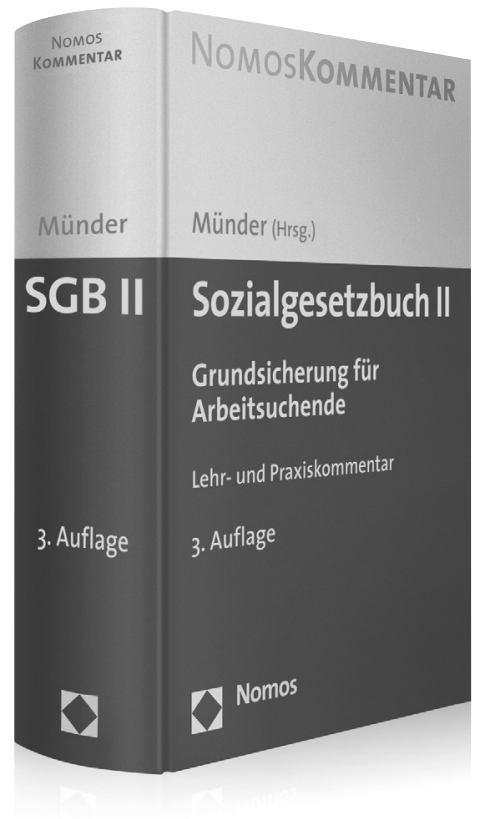

\section{Sozialgesetzbuch II \\ Grundsicherung für Arbeitsuchende \\ Lehr- und Praxiskommentar \\ Herausgegeben von Prof. Dr. Johannes Münder \\ 3. Auflage 2009, 919 S., geb., 49,- $€$, ISBN 978-3-8329-4639-5}

Der „Münder“ ist längst unverzichtbar geworden: Unklare Regelungen, ständige Änderungen durch den Gesetzgeber, offene Rechtsfragen und eine kaum noch überschaubare Anzahl von Gerichtsentscheidungen erschweren die Umsetzung des SGB II. Der neue LPK schafft Klarheit für die Rechtsanwendung, denn er bietet eine verständliche, praxisnahe und topaktuelle Kommentierung der gesetzlichen Vorschriften.

Der Lehr- und Praxiskommentar SGB II

- zeigt die exakten Voraussetzungen der Leistungsansprüche auf,

- berücksichtigt dabei die einschlägige Rechtsprechung des BSG und der Landessozialgerichte,

- liefert fundiertes Material zur Auslegung der unbestimmten Rechtsbegriffe,

- eröffnet inhaltliche Gestaltungsmöglichkeiten bei der Anwendung des Gesetzes und

- erleichtert so die Interessenvertretung der Leistungsberechtigten. 
"Da es nicht für alle reicht, springen die Armen ein." Ernst Bloch, deutscher Philosoph (1885-1977)

"Aber die Armen sind auf die Gerechtigkeit angewiesen, die Reichen sind auf die Ungerechtigkeit angewiesen, das entscheidet." Bertolt Brecht, deutscher Schriftsteller (1898-1956)

„Fürchten Sie sich vor den Armen? Warum nicht? "

Max Frisch, Fragebogen (1969)

"Ich war arm, ich war reich. Reich ist besser." Janis Joplin, amerikanische Rocksängerin (1943-1970)

"Reichtum ist besser als Armut.

Aber nur aus finanziellen Gründen."

Woody Allen, amerikanischer Regisseur und Schriftsteller (geb. 1935)

„In einem gut regierten Land ist Armut eine Schande, in einem schlecht regierten Reichtum. " Konfuzius, chinesischer Philosoph (551-479 v. Chr.)

"Wenn eine freie Gesellschaft den vielen, die arm sind, nicht helfen kann, kann sie auch die wenigen nicht retten, die reich sind." John F. Kennedy, amerikanischer Politiker (1917-1963) 